\title{
A Retrospective Study of Risk Factors for Symptomatic Anastomotic Leakage after Laparoscopic Anterior Resection of the Rectal Cancer without a Diverting Stoma
}

\author{
Zhi-Jie Wang $(\mathbb{D}$ and Qian Liu \\ Department of Colorectal Surgery, National Cancer Center/National Clinical Research Center for Cancer/Cancer Hospital, \\ Chinese Academy of Medical Sciences and Peking Union Medical College, Beijing 100021, China \\ Correspondence should be addressed to Qian Liu; qianliu_a123@163.com
}

Received 4 January 2020; Accepted 21 March 2020; Published 13 April 2020

Academic Editor: Roberto Caronna

Copyright ( 2020 Zhi-Jie Wang and Qian Liu. This is an open access article distributed under the Creative Commons Attribution License, which permits unrestricted use, distribution, and reproduction in any medium, provided the original work is properly cited.

\begin{abstract}
Background. Anastomotic leakage (AL) is a common and devastating postoperative issue for patients who have undergone anterior resection of rectal carcinoma and can lead to increased short-term morbidity and mortality. Moreover, it might be associated with a worse oncological prognosis of tumors. This study is aimed at exploring the risk factors for symptomatic AL after laparoscopic anterior resection (LAR) for rectal tumors without a preventive diverting stoma. Materials and Methods. This case control study retrospectively reviewed the data of 496 consecutive patients who underwent LAR of the rectum without a preventive diverting stoma at the Cancer Hospital, Chinese Academy of Medical Sciences between September 2016 and September 2017. All patients were divided into an AL group and a control group based on the occurrence of postoperative symptomatic AL. Factors regarding patient-related variables, operation-related variables, and tumor-related variables were collected and assessed between the two groups through univariate and multivariate logistic regression analyses to identify independent risk factors for AL. Results. In total, 18 (3.6\%) patients developed postoperative symptomatic AL. Univariate analysis showed that a synchronous primary malignancy of the left hemicolon $(P=0.047)$, intraoperative chemotherapy $(P=0.003)$, and level of anastomosis $(P=0.033)$ were significantly related with AL. Multivariate analysis was subsequently performed to adjust for confounding biases and confirmed that a synchronous primary malignancy of the left hemicolon (odds ratio (OR), 12.225; 95\% confidence interval (CI), 1.764-84.702; $P=0.011)$, intraoperative chemotherapy (OR, 3.931; 95\% CI, 1.334-11.583; $P=0.013$ ), and level of anastomosis (OR, 3.224; 95\% CI, 1.124-9.249; $P=0.030)$ were independent risk factors for symptomatic AL for patients who received LAR for rectal neoplasms without a preventive diverting stoma. Conclusions. Synchronous primary malignancy of the left hemicolon, intraoperative chemotherapy, and a low anastomotic level can increase the risks of postoperative symptomatic $\mathrm{AL}$ after LAR of the rectum without a protective diverting stoma.
\end{abstract}

\section{Introduction}

Anastomotic leakage (AL) is defined as a defect of the intestinal wall integrity at the anastomotic site, which leads to communication between the lumen of the bowel and the pelvic cavity (Figure 1). It is a major and serious surgical complication after anterior resection of rectal carcinoma, with the reported incidence varying considerably from $2.2 \%$ to $18.6 \%$ [1-3]. AL is associated with a prolonged hospital stay, increased medical costs, and a high occurrence of morbidity and mortality in a short time. Moreover, patients who developed AL can have poor long-term anorectal function resulting from pelvic fibrosis, which includes decreased maximum tolerated volume, increased fecal frequency, urgency, and incontinence [4]. More seriously, AL can also promote pelvic recurrence and decrease the overall survival, as it contributes to the spread of intraluminal residual tumor cells and local inflammatory related immunosuppression and delays postoperative adjuvant therapy $[5,6]$.

Patients with symptomatic AL usually present with symptoms and signs of fever, abdominal pain, peritonitis, and fecal discharge from the pelvic drainage [7]. Pelvic CT scans can show pneumatosis and hydrops around the 


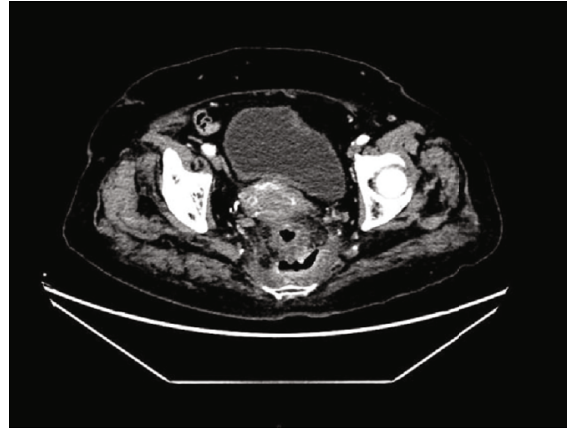

(a)

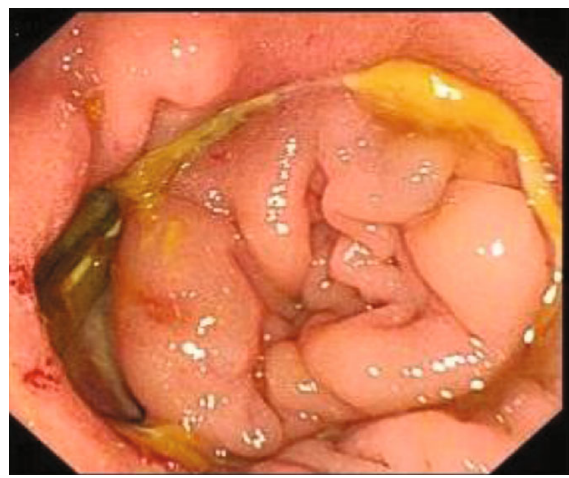

(c)

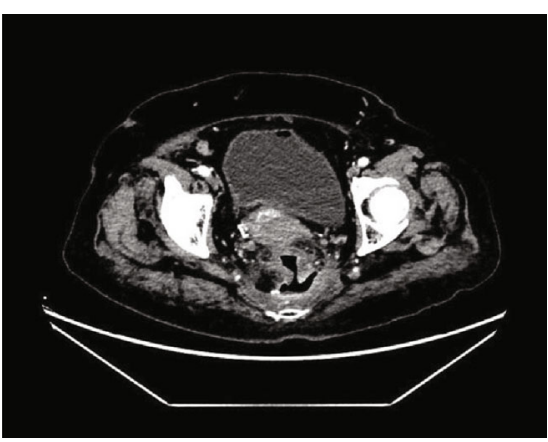

(b)

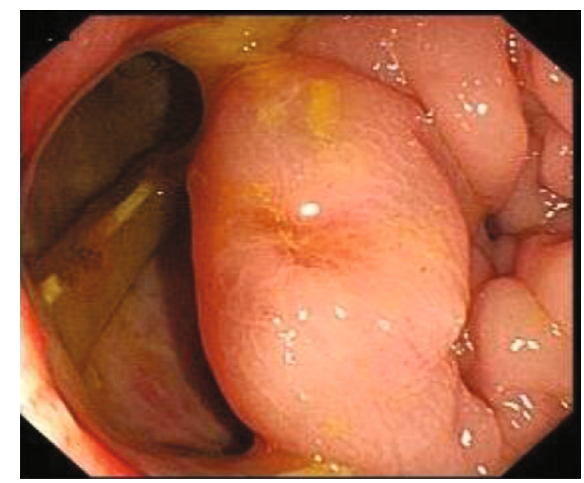

(d)

Figure 1: Images of anastomotic leakage. (a) Pelvic contrast-enhanced computed tomography picture showing pneumatosis and hydrops around the anastomosis; (b) pelvic contrast-enhanced computed tomography picture showing the communication between the lumen of the bowel and pelvic cavity; (c) endoscopic picture showing the defect of anastomosis; (d) endoscopic picture showing that the pelvic drainage tube can be seen from the anastomotic defect.

anastomosis in the pelvic cavity. Based on the proposal by the International Study Group of Rectal Cancer in 2010, rectal AL can be classified as grades A, B, and C. Patients with grade A AL need no medical interventions, patients with grade B AL need only conservative treatment, and patients who develop grade $\mathrm{C}$ AL require a secondary operation. Symptomatic AL includes both grade $\mathrm{B}$ and $\mathrm{C} \mathrm{AL}$ [8].

Many previous clinical studies have explored the risk factors and mechanisms of AL; however, most of these studies enrolled all patients who underwent laparotomy or laparoscopic surgery and all patients who received a protective stoma, which might lead to considerably different conclusions $[1,9,10]$. Laparoscopic surgery comprises a growing percentage of rectal surgeries and has been the main operation method in many countries and areas. Rectal surgery is usually difficult due to an insufficient operative view and limited working space in the pelvic cavity. The laparoscopic technique can provide a better operative field to facilitate surgery, but it also increases the difficulty of rectal transection because it is more difficult to provide adequate traction and effective cutting angles for the endolinear surgical stapler, unlike with open surgery. Therefore, the risk factors of AL may differ between laparotomy and laparoscopic surgery. Moreover, a protective diverting stoma can significantly prevent the occurrence of symptomatic AL [3]. Given the heterogeneity in rectal surgery resulting from different surgical methods and the creation of diverting stoma, we enrolled only patients who had received laparoscopic surgery without a diverting stoma to explore the risk factors for symptomatic AL.

\section{Materials and Methods}

2.1. Patients and Study Design. Our study was approved by the ethics committee of our institution and was conducted following the rules of the Helsinki Declaration of the World Medical Association. All patients in our study were diagnosed with rectal carcinoma through pathological biopsy and evaluation. We searched electronic medical records from the Cancer Hospital, Chinese Academy of Medical Sciences between September 2016 and September 2017. A total of 673 consecutive patients with rectal carcinoma had received anterior resection of the rectum at our center. After excluding 16 patients who had received laparotomy and 11 patients who had undergone conversion from laparoscopy to laparotomy, laparoscopic surgery was performed on 646 cases. Among these, 150 consecutive patients received a protective diverting stoma. Eventually, 496 consecutive patients who had undergone laparoscopic anterior resection (LAR) of rectal carcinoma without a diverting stoma were enrolled in our investigation (Figure 2). We noted that the term LAR usually referred to low anterior resection of the rectum in most previous publications, which may cause confusion here. Indeed, our study included both patients who received operations 


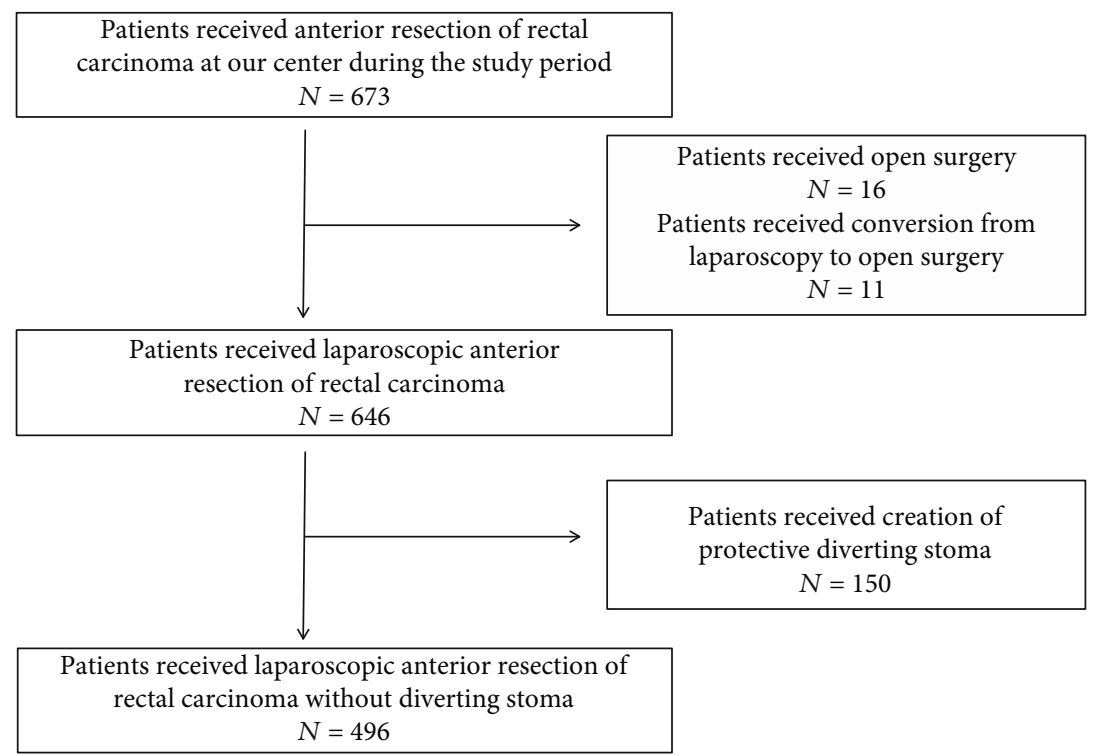

FIgURE 2: Flow chart presenting the patients' enrollment in our study.

above the peritoneal reflection and those who received operations below the reflection.

We retrospectively conducted a case control study in which patients who developed AL served in a case group while patients who did not were allocated to a control group. Variables regarding the demographic characteristics, living habits, comorbidities, nutritional status, preoperative chemoradiotherapy, intraoperative treatment, and tumor staging were carefully collected and analyzed between the two groups to explore the risk factors for postoperative AL.

2.2. Surgical Procedure. The bowel preparation was performed the day before surgery for each patient by oral administration of a sulfate-free polyethylene glycol electrolyte powder. In the operating room, the patients were placed in the lithotomy position; the pneumoperitoneum was established through intraperitoneal inflation with carbon oxide; and then the disconnection of vessels, lymph node dissection, and excision of the mesorectum were conducted through laparoscopic techniques. For patients with synchronous primary malignancy of the left hemicolon, extended resection with only one anastomosis was performed, and the resected intestine was usually longer than that of solitary rectal carcinoma. For patients who received natural orifice specimen extraction (NOSE) surgery, the specimens were extracted through the anus or vagina. For patients who received traditional LAR for the rectum, an additional small abdominal incision was created to extract the specimen. After extraction of the specimen, the pneumoperitoneum was established again, and the intestinal tract was reconstructed using a double stapling technique to form an end-to-end anastomosis. Peritoneal lavage was then routinely performed, and the air charging test was selectively carried out to evaluate the integrity of the anastomotic stoma for patients with a high risk of AL. For patients receiving intraoperative chemotherapy, the antineoplastic agents were then placed into the pelvic cavity. The available agents in our institution included lobaplatin and fluorouracil implants. Finally, one or two pelvic drainage tubes were inserted around the anastomotic stoma. For patients who were at high risk of postoperative $\mathrm{AL}$, such as neoadjuvant therapy, low level of anastomosis, tissue edema, and poor blood perfusion, a transanal tube might be placed to reduce intraluminal pressure.

2.3. Statistical Analysis. All data were described and analyzed using the Statistical Package for the Social Sciences (SPSS version 24.0; IBM Corp., Armonk, NY). Continuous data were described as the means \pm standard deviation (SD) and analyzed through a $t$-test when they were normally distributed. For continuous data that were not normally distributed, they were expressed as medians with interquartile ranges (IQRs) and further analyzed using Mann-Whitney $U$ tests. Both the categorical data and ordinal data were presented as the number of cases and percentages. Categorical data were analyzed using a $\chi^{2}$ test or Fisher's exact tests, while the ordinal data were subsequently analyzed using Mann-Whitney $U$ tests. All analyses were two-sided, and $P<0.05$ was regarded as statistically significant. To identify the independent risk factors for symptomatic AL, multivariate logistic regression analysis was performed, and variables with a $P$ value $<0.05$ in the univariate analysis and factors that were reported to promote AL in previous studies were included in this model.

\section{Results}

3.1. Postoperative AL. A total of 496 patients were included in our investigation, with a median age of 60 years (IQR 52-66). In total, 303 (61.1\%) were male and 193 (38.9\%) were female. Among the 496 patients, 18 (3.6\%) developed AL, and no grade A patient was enrolled in our study. Three $(16.7 \%)$ $\mathrm{AL}$ patients were classified as grade B, whereas $15(83.3 \%)$ were classified as grade $\mathrm{C}$ according to the proposal by the International Study Group of Rectal Cancer in 2010. All the 15 cases received a diverting stoma to control the abdominal 
infection; no cases received abdominoperitoneal resection. After that, 13 cases got their diverting stoma closed when the anastomotic stomas healed, while 2 cases did not due to their poor physical condition or anastomotic stenosis. In total, 17 (94.4\%) cases developed AL within a week after surgery and $1(5.6 \%)$ case occurred two months after the procedure (Table 1). No patients died during the perioperative period.

3.2. Patient-Related Variables. Patient-related variables are presented in Table 2. Patient demographics, living habits, comorbidities, neoadjuvant chemoradiotherapy, and nutritional status were compared and analyzed. We observed that AL was more likely to occur in rectal cancer patients who simultaneously suffered from a malignancy of the left hemicolon $(P=0.047)$. Other factors, including gender, age, body mass index, habits, preoperative chemoradiotherapy, and nutritional status, demonstrated no obvious association with AL.

3.3. Surgery-Related Variables. Surgery-related variables are presented in Table 3. All patients were treated with total mesorectal excision (TME). Most patients received traditional laparoscopic surgery in our study, and an additional incision was created for specimen extraction. One $(5.6 \%)$ and $32(6.7 \%)$ patients in the AL and control groups, respectively, underwent natural orifice specimen extraction (NOSE) surgery. NOSE surgery is an emerging surgical method in which resected specimens are extracted from the anus or vagina instead of from an auxiliary abdominal incision. The surgical approach exhibited no connection with AL occurrence. The AL group exhibited a greater proportion of patients treated by intraoperative chemotherapy compared to the control group (12 (66.7\%) in the AL group versus 159 $(33.3 \%)$ in the control group, $P=0.003)$. Moreover, it seemed that patients who had an anastomosis within $4 \mathrm{~cm}$ of the anal verge were at higher risk of $\operatorname{AL}(P=0.033)$. Statistical analysis revealed no obvious relationship between $\mathrm{AL}$ and operation time, reinforcing suture, intraoperative blood loss, perioperative transfusion, preservation of left colic artery, placement of the transanal tube, and number of stapler firings.

3.4. Neoplasm-Related Variables. Neoplasm-related variables are presented in Table 4 . Numerous previous reports have indicated that patients with low rectal carcinoma were more likely to develop AL; however, no significant relevance between AL and tumor location was observed in our study (all cases were regarded to be located above or below the peritoneal reflection based on intraoperative exploration in our study). No residual tumor was observed when pathologists examined the resected specimen in 26 patients, including 13 patients who underwent preoperative endoscopic resection and 13 patients who underwent preoperative chemotherapy or radiotherapy. AL occurred more frequently in stage T3/T4 tumors and in poorly differentiated cases, but this difference was not further confirmed in a subsequent statistical analysis.

3.5. Multivariate Analysis. Univariate analysis revealed that a synchronous primary malignancy of the left hemicolon $(P=0.047)$, intraoperative chemotherapy $(P=0.003)$, and
TABLE 1: AL patients.

AL patients $(n=18)$

\begin{tabular}{lc}
\hline AL grade, $n(\%)$ & $0(0 \%)$ \\
A & $3(16.7 \%)$ \\
B & $15(83.3 \%)$ \\
C & \\
Occurrence time of AL, $n(\%)$ & $17(94.4 \%)$ \\
Early AL & $1(5.6 \%)$ \\
Delayed AL
\end{tabular}

AL: anastomotic leakage.

the level of anastomosis $(P=0.033)$ were risk factors for AL. To adjust for confounding bias, we further enrolled these and other variables that were previously thought to increase the risks of AL in a subsequent multivariate analysis and confirmed that a synchronous primary malignancy of the left hemicolon (odds ratio (OR), 12.225; 95\% confidence interval (CI), 1.764-84.702; $P=0.011)$, intraoperative chemotherapy (OR, 3.931; 95\% CI, 1.334-11.583; $P=0.013$ ), and level of anastomosis (OR, 3.224; 95\% CI, 1.124-9.249; $P=0.030$ ) were independent risk factors for AL (Table 5).

\section{Discussion}

Numerous previous studies have explored the reasons for AL after anterior resection of rectal carcinoma and performed many measures to reduce its incidence, including preventive diverting stoma, intracorporeal reinforcing sutures, preservation of the left colonic artery, placement of a transanal tube, and mobilization of the splenic flexure of the colon to decrease the tension on the anastomosis [11-16]. Moreover, several scoring systems and prediction models have been built to predict the occurrence of AL [17-19]. However, AL remains the most common and devastating issue following anterior resection of the rectum, and the incidence and risk factors for AL varied considerably in previous reports. This is probably because most of these studies included patients who had received preventive diverting stoma or patients who had received laparotomy or laparoscopic surgery. Given that preventive diverting stomas can decrease the incidence of symptomatic AL and that laparoscopic surgery has been the main operation method in most countries and areas, our study included only patients who had undergone laparoscopic surgery without a diverting stoma. Finally, a synchronous primary malignancy of the left hemicolon, intraoperative chemotherapy, and level of anastomosis were confirmed to be associated with the occurrence of AL.

Synchronous colorectal carcinoma refers to the simultaneous detection of two or more colorectal malignant lesions in a single patient at the initial diagnosis. It accounts for $1.1-8.1 \%$ of colorectal cancer, but whether it could lead to a poorer prognosis compared to solitary colorectal carcinoma is controversial [20-22]. Most previous reports have focused on exploring its risk factors and clinicopathologic features, but its impacts on surgical options and postoperative complications are less well studied. Surgical approaches depend on the distribution of synchronous cancers. For patients with 
TABLE 2: Patient-related variables.

\begin{tabular}{|c|c|c|c|}
\hline Variables & $\mathrm{AL}(+)(n=18)$ & $\mathrm{AL}(-)(n=478)$ & $P$ \\
\hline \multicolumn{4}{|l|}{ Age (yr) } \\
\hline Median (IQR) & $56(50.75,60.5)$ & $60(52,66)$ & 0.297 \\
\hline Sex, $n(\%)$ & & & 0.139 \\
\hline Male & $14(77.8 \%)$ & $289(60.5 \%)$ & \\
\hline Female & $4(22.2 \%)$ & $189(39.5 \%)$ & \\
\hline $\mathrm{BMI}\left(\mathrm{kg} / \mathrm{m}^{2}\right.$, mean $\left.\pm \mathrm{SD}\right)$ & $24.0 \pm 3.3$ & $24.1 \pm 3.5$ & 0.853 \\
\hline Smoking, $n(\%)$ & $6(33.3 \%)$ & $135(28.2 \%)$ & 0.638 \\
\hline Alcohol, $n(\%)$ & $6(33.3 \%)$ & $104(21.8 \%)$ & 0.383 \\
\hline Hypertension, $n(\%)$ & $4(22.2 \%)$ & $128(26.8 \%)$ & 0.875 \\
\hline Ischemic heart disease, $n(\%)$ & $0(0 \%)$ & $15(3.1 \%)$ & 1.000 \\
\hline Diabetes, $n(\%)$ & $2(11.1 \%)$ & $54(11.3 \%)$ & 1.000 \\
\hline Hepatitis, $n(\%)$ & $1(5.6 \%)$ & $27(5.6 \%)$ & 1.000 \\
\hline History of malignancy, $n(\%)$ & $0(0 \%)$ & $17(3.6 \%)$ & 1.000 \\
\hline Synchronous primary malignancy of left hemicolon, $n(\%)$ & $2(11.1 \%)$ & $8(1.7 \%)$ & 0.047 \\
\hline Incomplete intestinal obstruction, $n(\%)$ & $2(11.1 \%)$ & $41(8.6 \%)$ & 1.000 \\
\hline Preoperative chemotherapy, $n(\%)$ & $3(16.7 \%)$ & $58(12.1 \%)$ & 0.834 \\
\hline Preoperative radiotherapy, $n(\%)$ & $1(5.6 \%)$ & $27(5.6 \%)$ & 1.000 \\
\hline Preoperative hemoglobin $(\mathrm{g} / \mathrm{L}$, mean $\pm \mathrm{SD})$ & $137.7 \pm 17.4$ & $137.3 \pm 17.3$ & 0.830 \\
\hline Preoperative albumin $(g / L$, mean $\pm S D)$ & $43.2 \pm 3.7$ & $44.2 \pm 3.6$ & 0.247 \\
\hline ASA grade, $n(\%)$ & & & 0.620 \\
\hline 1 & $0(0 \%)$ & $18(3.8 \%)$ & \\
\hline 2 & $17(94.4 \%)$ & $433(90.6 \%)$ & \\
\hline 3 & $1(5.6 \%)$ & $27(5.6 \%)$ & \\
\hline
\end{tabular}

AL: anastomotic leakage; IQR: interquartile range; SD: standard deviation; BMI: body mass index; ASA: American Society of Anesthesiologists.

TABLE 3: Surgery-related variables.

\begin{tabular}{|c|c|c|c|}
\hline Variables & $\mathrm{AL}(+)(n=18)$ & $\mathrm{AL}(-)(n=478)$ & $P$ \\
\hline Natural orifice specimen extraction surgery, $n(\%)$ & $1(5.6 \%)$ & $32(6.7 \%)$ & 1.000 \\
\hline Operation time (min, mean \pm SD) & $171.6 \pm 45.4$ & $162.5 \pm 62.4$ & 0.206 \\
\hline Consolidation suture, $n(\%)$ & $1(5.6 \%)$ & $71(14.9 \%)$ & 0.448 \\
\hline Intraoperative chemotherapy, $n(\%)$ & $12(66.7 \%)$ & $159(33.3 \%)$ & 0.003 \\
\hline Estimated blood loss $(\mathrm{mL}$, mean $\pm \mathrm{SD})$ & $56.7 \pm 34.0$ & $65.1 \pm 88.0$ & 0.506 \\
\hline Transfusion, $n(\%)$ & $1(5.6 \%)$ & $22(4.6 \%)$ & 0.641 \\
\hline Left colic artery preservation, $n(\%)$ & $1(5.6 \%)$ & $39(8.2 \%)$ & 1.000 \\
\hline Transanal tube, $n(\%)$ & $7(38.9 \%)$ & $270(56.5 \%)$ & 0.140 \\
\hline Anastomotic level from anal verge $(\mathrm{cm})$ & & & 0.033 \\
\hline$\leq 4$ & $11(61.1 \%)$ & $174(36.4 \%)$ & \\
\hline$>4$ & $7(39.9 \%)$ & $304(63.6 \%)$ & \\
\hline Number of stapler firing, $n(\%)$ & & & 0.819 \\
\hline 1 and 2 & $13(72.2 \%)$ & $370(7740 \%)$ & \\
\hline Greater than 2 & $5(27.8 \%)$ & $108(22.6 \%)$ & \\
\hline
\end{tabular}

AL: anastomotic leakage; SD: standard deviation.

synchronous carcinoma located at the rectum and left hemicolon, extended resection with only one anastomosis is the most common choice [23]. To date, only one report has demonstrated that synchronous colorectal carcinomas are a high risk factor for postoperative AL [24]. Our study included rectal cancer patients with synchronous cancer of the left hemicolon and found a significant association with AL. All of these patients underwent extended surgery, and the resected 
TABLE 4: Tumor-related variables.

\begin{tabular}{|c|c|c|c|}
\hline Variables & $\mathrm{AL}(+)(n=18)$ & $\operatorname{AL}(-)(n=478)$ & $P$ \\
\hline Tumor location, $n(\%)$ & & & 0.602 \\
\hline Above peritoneal reflection & $13(72.2 \%)$ & $383(80.1 \%)$ & \\
\hline Below peritoneal reflection & $5(27.8 \%)$ & $95(19.9 \%)$ & \\
\hline Pathological T stage, $n(\%)$ & & & 0.246 \\
\hline Tis, $\mathrm{T} 1, \mathrm{~T} 2$, and no tumor residual after preoperative therapy & $3(16.7 \%)$ & $140(29.3 \%)$ & \\
\hline $\mathrm{T} 3$ and $\mathrm{T} 4$ & $15(83.3 \%)$ & $338(70.7 \%)$ & \\
\hline Pathological N stage, $n(\%)$ & & & 0.854 \\
\hline N0 and no tumor residual after preoperative therapy & $10(55.6 \%)$ & $255(53.3 \%)$ & \\
\hline $\mathrm{N} 1$ and $\mathrm{N} 2$ & $8(44.4 \%)$ & $223(46.7 \%)$ & \\
\hline Pathological M stage, $n(\%)$ & & & 0.553 \\
\hline M0 & $18(100 \%)$ & $448(93.7 \%)$ & \\
\hline M1 & $0(0 \%)$ & $30(6.3 \%)$ & \\
\hline TNM stage, $n(\%)$ & & & 0.746 \\
\hline 0 -II and no tumor residual after preoperative therapy & $10(55.6 \%)$ & $247(51.7 \%)$ & \\
\hline III-IV & $8(44.4 \%)$ & $231(48.3 \%)$ & \\
\hline Degree of differentiation, $n(\%)$ & & & 0.337 \\
\hline Low, low-middle grade & $7(38.9 \%)$ & $136(28.5 \%)$ & \\
\hline Middle, high-middle, high grade, and no tumor residual after therapy & $11(61.1 \%)$ & $342(71.5 \%)$ & \\
\hline
\end{tabular}

AL: anastomotic leakage.

TABLE 5: Multivariate logistic regression analysis.

\begin{tabular}{lccc}
\hline Variables & $P$ & OR & 95\% CI \\
\hline Gender & 0.095 & 2.742 & $0.841-8.943$ \\
Age & 0.723 & 1.009 & $0.962-1.057$ \\
BMI & 0.644 & 1.033 & $0.899-1.188$ \\
Diabetes & 0.721 & 1.332 & $0.276-6.421$ \\
Synchronous primary malignancy of left hemicolon & 0.011 & 12.225 & $1.764-84.702$ \\
Preoperative radiotherapy & 0.753 & 1.415 & $0.163-12.282$ \\
Intraoperative chemotherapy & 0.013 & 3.931 & $1.334-11.583$ \\
Perioperative transfusion & 0.400 & 2.561 & $0.287-22.887$ \\
Level of anastomosis & 0.030 & 3.224 & $1.124-9.249$ \\
Transanal tube & 0.171 & 0.486 & $0.173-1.364$ \\
Number of stapler firing & 0.852 & 0.895 & $0.279-2.868$ \\
\hline
\end{tabular}

BMI: body mass index; OR: odds ratio; CI: confidence interval.

intestine was usually longer than that of solitary rectal carcinoma, which might increase the tension of the anastomoses and promote the occurrence of AL.

For patients with locally advanced rectal carcinoma, neoadjuvant therapy has been accepted as an important treatment modality to improve the clinical outcomes. However, we observed that only $18 \%$ of our patients received this therapy, which might be a result of poor economic conditions and fear of side effects. Intraoperative chemotherapy has been gradually performed in rectal cancer patients in recent years, aimed at decreasing the rate of local recurrence and distant metastasis [25]. It is generally indicated for patients with $\mathrm{T} 3 / \mathrm{T} 4$ or $\mathrm{N}+$ rectal cancer. It is conducted by placing antitumor agents into the pelvic cavity to eradicate the resid- ual cancer cells at the end of the operation. Previous reports have confirmed that this emerging treatment modality can improve the oncologic prognosis of patients with rectal carcinoma, but its impacts on postoperative AL remain controversial [26]. Our study presented a higher proportion of patients exposed to intraoperative chemotherapy in AL patients than in non-AL patients $(66.7 \%$ in the AL group versus $33.3 \%$ in the non-AL group), which was a significant difference in both the univariate and multivariate analyses This implies that intraoperative chemotherapy might be a risk factor for postoperative AL in rectal surgery. Although there have been few clinical studies on the relationship between intraoperative chemotherapy and AL, many animal studies have indicated that intraoperative chemotherapy can lead to the occurrence 
of AL in rats [27]. The rapid proliferation of regenerative cells is essential during the healing process of intestinal anastomoses, but implantation of antineoplastic agents can inhibit their proliferation. These agents can inhibit the activity of fibroblasts and decrease the deposition of collagen, which can reduce the mechanical strength of the anastomoses [28]. Moreover, intraoperative chemotherapy can suppress the process of vascularization, promote oxidative stress, and enhance the inflammatory response, which can contribute to anastomotic tissue necrosis $[29,30]$. Surgeons need to carefully evaluate the risks of AL for patients before conducting this treatment.

Rectal cancer surgery is generally more difficult compared with colon cancer surgery given the insufficient operative view and limited working space in the pelvic cavity. The laparoscopic technique can provide an obviously better operative field than traditional laparotomy, but it also increases the difficulty of rectal transection and normally requires more stapler firings, as the cutting angle of the endolinear stapler is ineffective in this technique, and this difficulty is significantly increased when the rectum is resected at a low level [31]. Therefore, the anastomotic level has been regarded as a risk factor for AL in many previous studies, but the best cutoff value differed across reports [32]. In one systematic review that enrolled 4580 patients, the incidence of AL for patients with anastomosis levels below $5 \mathrm{~cm}$ from the anal verge was 8.3 times greater than the incidence for those above $5 \mathrm{~cm}$ [33]. In another retrospective report, the cutoff value was set as $4 \mathrm{~cm}$ and the AL rate was 6 times higher when the anastomosis was located within $4 \mathrm{~cm}$ of the anal verge [34]. In our study, we observed that an anastomosis level $\leq 4 \mathrm{~cm}$ was significantly associated with the occurrence of AL (5.9\% versus 2.3\%). This can be attributed to the increased difficulties in performing the technique as the anastomotic distance from the anal verge decreases. In addition, a poorer blood supply caused by a lower anastomotic level might be another reason for the high risks of $\mathrm{AL}$. We also explored the relationship between $\mathrm{AL}$ and tumor location, but no statistically significant differences were observed, which was in line with some previous reports [35]. Given the difference about the length of the distal margin from the low border of the rectal tumor between tumors located above and tumors located below the peritoneal reflection, we believe the level of anastomosis may be a better index to predict AL than tumor location.

Given the serious consequence of AL, most surgeons tend to choose the creation of a preventive diverting stoma for patients with high risks of AL. Our study excluded 150 (22.3\%) patients who received a diverting stoma; it is unclear how many of them truly benefited from this procedure. Many of them may not develop AL even if they do not have a prophylactic stoma, and it brings patients risks of stoma-related complications and a second operation to close it. Surgeons need to think carefully about this for patients. Neoadjuvant therapy, low level of anastomosis, tissue edema, and poor blood perfusion are mostly believed to lead to AL. In our center, patients with two or more of these risk factors need to receive a diverting stoma. Moreover, for patients with risky distrustful anastomoses, such as a positive result of air charg- ing test and incomplete incisal margin from staplers, they will undergo protective stomas even if no risk factors exist.

Our study has the following limits. First, the retrospective nature of our study makes the bias from patient selection and data collection difficult to avoid. Second, the incidence of AL in our report is much lower than that in most previous reports, and the limited number of patients in the AL group might hinder the findings of more risk factors for symptomatic AL in our study.

\section{Conclusions}

In conclusion, our present research determined that synchronous primary malignancy of the left hemicolon, intraoperative chemotherapy, and low anastomotic levels were independent risk factors for symptomatic AL after LAR for rectal carcinoma.

\section{Data Availability}

The data used to support the findings of this study are available from the corresponding author upon request.

\section{Conflicts of Interest}

The authors declare that there is no conflict of interest with regard to this research.

\section{Authors' Contributions}

Zhi-Jie Wang collected and analyzed the data and drafted the article; Qian Liu designed the research and revised the article.

\section{Acknowledgments}

This study is supported by the following grants: the National Key Research and Development Program/Prevent and Control Research for Important Non-Communicable Diseases (No. 2019YFC1315705), the Medicine and Health Technology Innovation Project of the Chinese Academy of Medical Sciences (No. 2017-12M-1-006), and the Special Fund of China Cancer Research Foundation/Beijing Hope Marathon (No. LC2017L03).

\section{References}

[1] J. Tanaka, T. Nishikawa, T. Tanaka et al., "Analysis of anastomotic leakage after rectal surgery: a case-control study," Annals of Medicine and Surgery, vol. 4, no. 2, pp. 183-186, 2015.

[2] B. C. Olsen, S. T. Sakkestad, F. Pfeffer, and A. Karliczek, "Rate of anastomotic leakage after rectal anastomosis depends on the definition: pelvic abscesses are significant," Scandinavian Journal of Surgery, vol. 108, no. 3, pp. 241-249, 2019.

[3] G. Salamone, L. Licari, A. Agrusa et al., "Usefulness of ileostomy defunctioning stoma after anterior resection of rectum on prevention of anastomotic leakage a retrospective analysis," Annali Italiani di Chirurgia, vol. 87, pp. 155-160, 2016.

[4] B. D. Shogan, E. M. Carlisle, J. C. Alverdy, and K. Umanskiy, "Do we really know why colorectal anastomoses leak?," 
Journal of Gastrointestinal Surgery, vol. 17, no. 9, pp. 16981707, 2013.

[5] S. Wang, J. Liu, S. Wang, H. Zhao, S. Ge, and W. Wang, "Adverse effects of anastomotic leakage on local recurrence and survival after curative anterior resection for rectal cancer: a systematic review and meta-analysis," World Journal of Surgery, vol. 41, no. 1, pp. 277-284, 2017.

[6] G. T. Noh, Y. S. Ann, C. Cheong et al., "Impact of anastomotic leakage on long-term oncologic outcome and its related factors in rectal cancer," Medicine, vol. 95, no. 30, article e4367, 2016.

[7] J. A. Yun, Y. B. Cho, Y. A. Park et al., "Clinical manifestations and risk factors of anastomotic leakage after low anterior resection for rectal cancer," ANZ Journal of Surgery, vol. 87, no. 11, pp. 908-914, 2017.

[8] N. N. Rahbari, J. Weitz, W. Hohenberger et al., "Definition and grading of anastomotic leakage following anterior resection of the rectum: a proposal by the International Study Group of Rectal Cancer," Surgery, vol. 147, no. 3, pp. 339-351, 2010.

[9] Q. Qin, T. Ma, Y. Deng et al., "Impact of preoperative radiotherapy on anastomotic leakage and stenosis after rectal cancer resection: post hoc analysis of a randomized controlled trial," Diseases of the Colon and Rectum, vol. 59, no. 10, pp. 934942, 2016.

[10] C. W. Kim, S. J. Baek, H. Hur, B. S. Min, S. H. Baik, and N. K. Kim, "Anastomotic leakage after low anterior resection for rectal cancer is different between minimally invasive surgery and open surgery," Annals of Surgery, vol. 263, no. 1, pp. 130-137, 2016.

[11] W.-T. Zhao, N.-N. Li, D. He, and J.-Y. Feng, “Transanal tube for the prevention of anastomotic leakage after rectal cancer surgery: a systematic review and meta-analysis," World Journal of Surgery, vol. 41, no. 1, pp. 267-276, 2017.

[12] F.-G. Wang, W.-M. Yan, M. Yan, and M.-M. Song, “Comparison of anastomotic leakage rate and reoperation rate between transanal tube placement and defunctioning stoma after anterior resection: a network meta-analysis of clinical data," European Journal of Surgical Oncology, vol. 45, no. 8, pp. 13011309, 2019.

[13] Y. Cui, S. Sun, Z. Li, and W. Wang, "Safety, survival, and efficacy of preserving left colonic artery in rectal cancer surgery: a meta-analysis and review," Journal of Laparoendoscopic \& Advanced Surgical Techniques Part A, vol. 29, no. 11, pp. 1405-1413, 2019.

[14] T. Hinoi, M. Okajima, M. Shimomura et al., "Effect of left colonic artery preservation on anastomotic leakage in laparoscopic anterior resection for middle and low rectal cancer," World Journal of Surgery, vol. 37, no. 12, pp. 2935-2943, 2013.

[15] K. Maeda, H. Nagahara, M. Shibutani et al., "Efficacy of intracorporeal reinforcing sutures for anastomotic leakage after laparoscopic surgery for rectal cancer," Surgical Endoscopy, vol. 29, no. 12, pp. 3535-3542, 2015.

[16] J. Zeng and G. Su, "High ligation of the inferior mesenteric artery during sigmoid colon and rectal cancer surgery increases the risk of anastomotic leakage: a meta-analysis," World Journal of Surgical Oncology, vol. 16, no. 1, p. 157, 2018.

[17] T. Watanabe, H. Miyata, H. Konno et al., "Prediction model for complications after low anterior resection based on data from 33,411 Japanese patients included in the National Clinical Database," Surgery, vol. 161, no. 6, pp. 1597-1608, 2017.

[18] I. S. Reynolds, M. R. Boland, F. Reilly et al., "C-reactive protein as a predictor of anastomotic leak in the first week after ante- rior resection for rectal cancer," Colorectal Disease, vol. 19, no. 9, pp. 812-818, 2017.

[19] A. Arezzo, M. Migliore, P. Chiaro et al., "The REAL (REctal Anastomotic Leak) score for prediction of anastomotic leak after rectal cancer surgery," Techniques in Coloproctology, vol. 23, no. 7, pp. 649-663, 2019.

[20] A. K. Lam, S. S. Chan, and M. Leung, "Synchronous colorectal cancer: clinical, pathological and molecular implications," World Journal of Gastroenterology, vol. 20, no. 22, pp. 68156820, 2014.

[21] T. Kato, S. Alonso, Y. Muto et al., "Clinical characteristics of synchronous colorectal cancers in Japan," World Journal of Surgical Oncology, vol. 14, no. 1, p. 272, 2016.

[22] C. C. Chin, Y. H. Kuo, and J. M. Chiang, "Synchronous colorectal carcinoma: predisposing factors and characteristics," Colorectal Disease, vol. 21, no. 4, pp. 432-440, 2019.

[23] B. C. Lee, C. S. Yu, J. Kim et al., "Clinicopathological features and surgical options for synchronous colorectal cancer," Medicine, vol. 96, no. 9, article e6224, 2017.

[24] N. J. van Leersum, A. G. Aalbers, H. S. Snijders et al., "Synchronous colorectal carcinoma: a risk factor in colorectal cancer surgery," Diseases of the Colon and Rectum, vol. 57, no. 4, pp. 460-466, 2014.

[25] R. X. Zhang, J. Z. Lin, J. Lei et al., "Safety of intraoperative chemotherapy with 5-FU for colorectal cancer patients receiving curative resection: a randomized, multicenter, prospective, phase III IOCCRC trial (IOCCRC)," Journal of Cancer Research and Clinical Oncology, vol. 143, no. 12, pp. 25812593, 2017.

[26] H. Yuan, B. Zheng, and S. Tu, "Clinical research of intraperitoneal implantation of sustained-release 5-fluorouracil in advanced colorectal cancer," World Journal of Surgical Oncology, vol. 13, no. 1, p. 320, 2015.

[27] A. Aghayeva, C. Benlice, I. A. Bilgin et al., "The effects of hyperthermic intraperitoneal chemoperfusion on colonic anastomosis: an experimental study in a rat model," Tumori, vol. 103, no. 3, pp. 307-313, 2017.

[28] D. Kanellos, M. G. Pramateftakis, I. Mantzoros et al., "The effects of the intraperitoneal administration of oxaliplatin and 5-FU on the healing of colonic anastomoses: an experimental study," Techniques in Coloproctology, vol. 15, Supplement 1, pp. 111-115, 2011.

[29] M. G. Pramateftakis, D. Kanellos, I. Mantzoros et al., "Intraperitoneally administered irinotecan with 5-fluorouracil impair wound healing of colonic anastomoses in a rat model: an experimental study," Techniques in Coloproctology, vol. 15, Supplement 1, pp. 121-125, 2011.

[30] S. Arapoglou, A. Kambaroudis, I. Grivas et al., "The effect of iloprost in the healing of colonic anastomosis in rats under chemotherapy with irinotecan," Chirurgia, vol. 112, no. 6, pp. 705-713, 2017.

[31] D. H. Choi, J. K. Hwang, Y. T. Ko et al., "Risk factors for anastomotic leakage after laparoscopic rectal resection," Journal of the Korean Society of Coloproctology, vol. 26, no. 4, pp. 265273,2010

[32] Y. Liu, X. Wan, G. Wang et al., "A scoring system to predict the risk of anastomotic leakage after anterior resection for rectal cancer," Journal of Surgical Oncology, vol. 109, no. 2, pp. 122-125, 2014.

[33] H. Qu, Y. Liu, and D. S. Bi, "Clinical risk factors for anastomotic leakage after laparoscopic anterior resection for rectal 
cancer: a systematic review and meta-analysis," Surgical Endoscopy, vol. 29, no. 12, pp. 3608-3617, 2015.

[34] L. Wang and J. Gu, "Risk factors for symptomatic anastomotic leakage after low anterior resection for rectal cancer with 30 Gy/10 f/2 w preoperative radiotherapy," World Journal of Surgery, vol. 34, no. 5, pp. 1080-1085, 2010.

[35] S. Shinji, Y. Ueda, T. Yamada et al., "Male sex and history of ischemic heart disease are major risk factors for anastomotic leakage after laparoscopic anterior resection in patients with rectal cancer," BMC Gastroenterology, vol. 18, no. 1, p. 117, 2018. 